\title{
PEDIATRIC CORONAVIRUS DISEASE 2019: HOW TO ASSESS CHEST DISEASE?
}

\author{
Adele Corcione $^{1}$, Federica Annunziata ${ }^{2}$, Melissa Borrelli $^{2}$, and Francesca Santamaria ${ }^{2}$ \\ ${ }^{1}$ Federico II University Hospital \\ ${ }^{2}$ Federico II University
}

May 6, 2020

\begin{abstract}
The severe acute respiratory syndrome coronavirus 2 (SARS-CoV-2) has emerged as a serious health problem worldwide. In the pediatric population, currently available epidemiological data seem reassuring as the incidence of coronavirus disease 2019 (COVID-19) is much lower than in adults, with less critical cases and very few deaths. At present, there are no evidence-based studies on chest imaging in pediatric COVID-19. Chest X-rays showed non-specific findings and chest computed tomography (CT) exhibited similar, but fairly less severe CT changes compared to adult. Moreover, in approximately $50 \%$ of pediatric patients no correlation was found between chest CT imaging results and clinical characteristics. Lung ultrasound is rarely used, despite its unquestionable benefits as it can be performed at bed-side with a portable device, which minimizes virus transmission, is cheap and can be easily repeated. In conclusion, the chest imaging use in children, who are typically spared from severe infection, deserve recommendations different than adults also considering the increased risk of radiations exposure. In view of this, pediatric comparative studies among different chest imaging techniques, either less or more invasive, are urgently needed possibly after standardization of interpretation criteria of lung ultrasound.
\end{abstract}

\section{To the Editor,}

The number of cases with coronavirus disease 2019 (COVID-19) caused by severe acute respiratory syndrome coronavirus 2 (SARS-CoV-2) is dramatically increasing, with high fatality rate. The medical community has mixed feelings about pediatric COVID-19. On the one hand, the epidemiologic reports seem reassuring as retrospective data show that most children have mild disease or are asymptomatic, there are fairly less critical cases than adults and few deaths. In Italy, of all infected, $1.9 \%$ had less than 19 years, with $0.1 \%$ mortality rate $(99.9 \% \text { among adults })^{1}$, On the other hand, despite the bulk of publications, many aspects of pediatric COVID-19 are blurry, and in particular how evaluating pulmonary involvement in children infected from SARS-CoV-2 is not completely defined. In a recent issue ofPediatric Pulmonology, Zhu et al from China reported the clinical characteristics of a case series of 10 children or adolescents with COVID-19 who were admitted with fever $(40 \%)$ or cough $(30 \%)$ or headache $(20 \%)$, and no fatigue or shortness of breath ${ }^{2}$. Thirty percent were asymptomatic. All had normal white blood cells or acute phase reactants. In all cases, chest imaging included computed tomography (CT), but not conventional X-rays (CXR) or lung ultrasound (US). Five out 10 cases had negative CT, although fever and/or cough were present in 3 children, while bilateral or unilateral ground-glass opacities (GGO) were detected in the remaining 5 patients, either in the absence or presence of symptoms ( 2 and 3 cases, respectively). These data prompted us to some considerations on the use of chest imaging in children with confirmed COVID-19 requiring lung disease assessment.

At present, there are no evidence-based studies on chest imaging in pediatric COVID-19. Some authors from China reported that either in the presence or absence of symptoms, CXR findings may be non-specific, and that chest CT might support information in early disease ${ }^{3}$. Compared to adults, children exhibited similar, but fairly less severe CT changes, ranging from no anomalies at all (20-65\%) to unilateral (21-30\%) 
or bilateral (10-50\%) changes, with predominantly sub-pleural distribution ${ }^{3}$. Typical findings are patchy shadows, ground-glass opacities (GGO), consolidations with halo sign and tiny nodules ${ }^{2,3}$. More diffuse CT changes, including bilateral consolidations may be observed in children with co-infections, in those aged less $<3$ years or requiring intensive care ${ }^{3}$. In inpatients, chest CT may also be used to follow the evolution of lung changes and assess the recovery of abnormalities, or progression of consolidations into GGO, or residual fibrotic bands. However, the demonstration of either GGO in the absence of symptoms, or of normal CT in children with mild symptoms, raises doubts on use of CT in pediatric COVID-19².

Actually, the British Paediatric Respiratory Society recommended not to perform any chest imaging routinely in children, even in those who require little supplemental oxygen on admission ${ }^{4}$. Conversely, a China document advised that suspected or confirmed cases should undertake chest X-ray examination as soon as possible ${ }^{3}$. The British Paediatric Respiratory Society recommended that chest X-rays, possibly with a portable device, is done only in children requiring prolonged oxygen on day 3 of admission or non-invasive ventilation, or if there is a specific clinical question ${ }^{4}$. For avoiding excessive radiation exposure and contamination of suites, personnel and equipment, chest CT should be reserved to unstable cases with increasingly clinical deterioration, or if surgery cannot be postponed. In light of this, using US as radiation-free alternative seems interesting, also taking into account the predilection for the disease in the subpleural regions.

In a large pediatric COVID-19 population from Italy, the imaging technique of choice was CXR (obtained in $35 \%$ of cases), chest CT was not performed, and bedside LUS, done only if an expert sonographer was available, was done in $10 \%$ of the cases as alternative option to CXR or to confirm negative CXR despite severe symptoms ${ }^{5}$. Of all patients, $21 \%$ were asymptomatic, and $58 \%, 20 \%$, and $1 \%$ had mild or moderateto-severe or critical COVID-19, respectively. Eleven children showed lung involvement at CXR or US in the absence of hypoxia, likely because of early hospital admission. Although it cannot be ruled-out that the definition of moderate-to-severe disease in $20 \%$ of cases was achieved because CXR, and not CT was used, actually CT may overestimate the impact of chest-associated pediatric COVID-19, yet excessive use of radiation should prompt to search for alternate, less invasive techniques as demonstrated in pediatric lung disorders $^{6}$. Benefits of lung US in pediatric COVID-19 are unquestionable as it can be performed at bedside with a portable device, which minimizes virus transmission, is cheap and can be repeated. Though, the availability of experts, as well the absence of standardized interpretation criteria, greatly limits its routine application.

Currently, no recommendations are available on the chest imaging techniques of choice in the assessment of lung disease in pediatric COVID-19. The Table summarizes the chest imaging findings from pediatric studies.

Actually, children and adolescents, who are typically spared from severe infection, deserve recommendations different than adults, with special consideration to the risk of radiations exposure which is significantly increased among children. In view of this, pediatric comparative studies among different chest imaging techniques, either less or more invasive, are urgently needed in pediatric COVID-19, possibly after standardization of interpretation criteria (for instance, for US). As residual lung fibrosis may develop after viral infections also in children with COVID-19, a longitudinal follow-up study with invasive or less invasive imaging techniques would be of remarkable value. Finally, chest imaging findings might also be used as measure of efficacy of old or novel therapies in adult as well in pediatric COVID-19.

There is an urgent need to understand several issues of pediatric COVID-19. First and foremost, while waiting for vaccine, no one should lower the guard in preventing the spreading of the virus from asymptomatic or presymptomatic children, reducing the proportion of those infected, providing them with the best management also including imaging techniques for lung disease extension and severity evaluation, and thus keeping under control the increase of cases and deaths from this outbreak, likely the most dramatic ever lived by humans over the last century.

\section{References}

1. Istituto Superiore di Sanità. Sorveglianza Integrata COVID-19 in Italia. Available 
from:https://www.epicentro.iss.it/coronavirus/bollettino/Infografica_4(Accessed on May 5, 2020)

2. Zhu L, Wang J, Huang R, Liu L, Zhao H, Wu C, Zhu C. Clinical characteristics of a case series of children with coronavirus disease 2019. Pediatr Pulmonol. 2020;1-3.

3. Shen K, Yang Y, Wang T, Zhao D, Jiang Y, Jin R, Zheng Y, Xu B, Xie Z, Lin L, et al. Diagnosis, treatment, and prevention of 2019 novel coronavirus infection in children: experts' consensus statement. World J Pediatr. 2020 Feb 7. doi: 10.1007/s12519-020-00343-7. [Epub ahead of print]

4. British Paediatric Respiratory Society. Management of children admitted to hospital with COVID-19 infection (Version 2). Available from:https://www.rcpch.ac.uk/resources/covid-19-guidance-paediatricservices (Accessed on May 5, 2020).

5. Parri N, Lenge M, Buonsenso D. Coronavirus Infection in Pediatric Emergency Departments (CONFIDENCE) Research Group. Children with Covid-19 in Pediatric Emergency Departments in Italy. N Engl J Med. 2020 May 1. doi: 10.1056/NEJMc2007617. Online ahead of print.

6. Montella S, Maglione M, Bruzzese D, Mollica C, Pignata C, Aloj G, Manna A, Esposito A, Mirra V, Santamaria F. Magnetic resonance imaging is an accurate and reliable method to evaluate non-cystic fibrosis paediatric lung disease. Respirology. 2012;17(1): 87-91. 